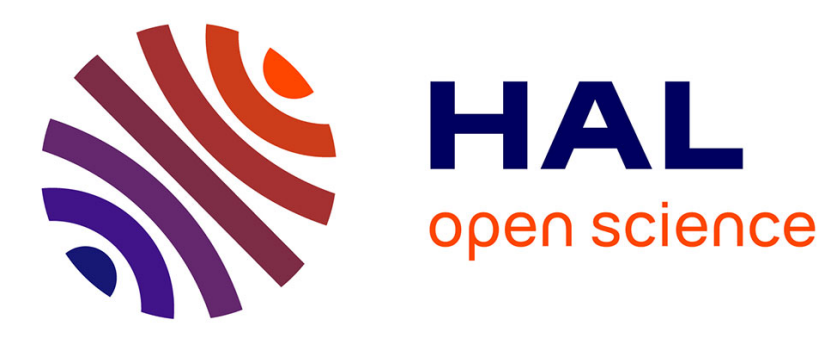

\title{
In Situ Fluorescence Tomography Enables a 3D Mapping of Enzymatic O 2 Reduction at the Electrochemical Interface
}

\author{
Bastien Tassy, Alice Dauphin, Hiu Mun Man, Hugo Le Guenno, Elisabeth \\ Lojou, Laurent Bouffier, Anne de Poulpiquet
}

\section{To cite this version:}

Bastien Tassy, Alice Dauphin, Hiu Mun Man, Hugo Le Guenno, Elisabeth Lojou, et al.. In Situ Fluorescence Tomography Enables a 3D Mapping of Enzymatic O 2 Reduction at the Electrochemical Interface. Analytical Chemistry, 2020, 92 (10), pp.7249-7256. 10.1021/acs.analchem.0c00844 . hal02936237

\section{HAL Id: hal-02936237 \\ https://hal.science/hal-02936237}

Submitted on 3 Dec 2020

HAL is a multi-disciplinary open access archive for the deposit and dissemination of scientific research documents, whether they are published or not. The documents may come from teaching and research institutions in France or abroad, or from public or private research centers.
L'archive ouverte pluridisciplinaire $\mathbf{H A L}$, est destinée au dépôt et à la diffusion de documents scientifiques de niveau recherche, publiés ou non, émanant des établissements d'enseignement et de recherche français ou étrangers, des laboratoires publics ou privés. 


\title{
In situ fluorescence tomography enables a 3D mapping of enzymatic $\mathrm{O}_{2}$-reduction at the electrochemical interface
}

\author{
Bastien Tassy, ${ }^{\dagger}$ Alice L. Dauphin, ${ }^{\dagger}$ Hiu Mun Man,${ }^{\dagger}$ Hugo Le Guenno, ${ }^{+}$Elisabeth Lojou, ${ }^{\dagger}$ Laurent \\ Bouffier, ${ }^{\ddagger}$ and Anne de Poulpiquet ${ }^{\dagger} * *$ \\ ${ }^{\dagger}$ Bioenergetics and Protein Engineering, UMR 7281, Aix-Marseille Univ., CNRS, Marseille, France \\ * Institute of Molecular Sciences, UMR 5255, Univ. Bordeaux, CNRS, Bordeaux INP, F-33400 Talence, France \\ ${ }^{+}$Microscopy facility, FR 3479, Mediterranean Institute of Microbiology, CNRS, Marseille, France
}

\begin{abstract}
Getting information about the fate of immobilized biomolecules and the evolution of their environment during turnover is a mandatory step towards bioelectrode optimization for effective use in biodevices. We demonstrate here the proof-ofprinciple characterization of the reactivity at an enzymatic electrode thanks to fluorescence confocal laser scanning microscopy (FCLSM) implemented in situ during the electrochemical experiment. The enzymatic $\mathrm{O}_{2}$-reduction involves proton and electron transfers. Therefore, fluorescence variation of a $\mathrm{pH}$-dependent fluorescent dye in the electrode vicinity enables the reaction visualization. Simultaneous collection of electrochemical and fluorescence signals gives valuable space- and time-resolved information. Once the technical challenges of such a coupling are overcome, in situ FCLSM affords a unique way to explore reactivity at the electrode surface and in the electrolyte volume. Unexpected features are observed, especially the $\mathrm{pH}$ evolution of the enzyme environment, which is also indicated by a characteristic concentration profile within the diffusion layer. This coupled approach gives also access to a cartography of the electrode surface response (i.e. heterogeneity), which cannot be obtained solely by an electrochemical mean.
\end{abstract}

\section{INTRODUCTION}

Redox enzymes that catalyze electron transfer (ET) reactions in living organisms are highly relevant biomolecular tools. They feature unique properties that can be implemented in biotechnological devices, especially bioelectrochemical ones: extreme affinity and specificity towards their substrate, very high catalytic turnover and low overpotentials with respect to their substrate, bioavailability, biodegradability... In most bioelectrochemical devices, enzymes are immobilized at the electrode surface to exchange the electrons involved in the electrochemical reactions. Due to the large size of enzyme molecules, and the anisotropy of their electronic properties and surface characteristics, efficient immobilization at the electrochemical interface is not trivial and must be conducted and characterized carefully. Electrochemical methods provide a great amount of information about the characteristics of the enzyme itself, including its different redox states within catalysis, its immobilization at the electrode and the efficiency of the entire bioelectrochemical devices. However, they also have several intrinsic limitations, which justify the necessity to combine electrochemistry with other physical characterizations. Infrared spectroscopy (SEIRA, PM-IRRAS...) has been historically used to characterize the enzyme conformation or orientation when immobilized at the surface. ${ }^{1-2}$ This latter parameter is essential to enable a direct electronic wiring between the enzyme and the electrode. Electrochemical SPR (surface plasmon resonance) and QCM (quartz crystal microbalance) have been used to quantify the amount of enzyme immobilized at the electrode, ${ }^{3-6}$ which is hardly obtained solely by electrochemical methods. ${ }^{7}$ However, in all these methods, the signal output is averaged over the entire electrode surface area and therefore no data about local activity, inhibition or possible heterogeneities of enzyme immobilization is provided. To get spatially resolved information about orientation, activity, localization etc., different microscopy techniques have been implemented. ${ }^{8-10}$ Scanning probe microscopies (AFM, STM, SECM) were the most widely used techniques. By contrast, fluorescence microscopy has been very much underutilized, and the few examples found in the literature only report the characterization of dry samples to locate the enzyme in a polymer matrix, a carbon fiber, or a microstructured gold electrode. ${ }^{11-15}$

On the other hand, the basis for the coupling between electrochemistry and fluorescence microscopy in the abiotic context has been set in the early 1990's. ${ }^{16-18}$ Using fluorescein as a pHsensitive fluorophore, Engstrom and coworkers imaged water electrolysis at various electrode materials ${ }^{16}$ and studied in particular the reduction of oxygen. ${ }^{17}$ The ability of the technique to map local variations in electrochemical reactivity was exemplified with ET kinetics measurement at bimetallic $\mathrm{Ag} / \mathrm{Pt}$ electrodes as well as size and localization of silver deposits on glassy carbon (GC). They also extended the technique to anodic reactions that consume hydroxide ions by using quinine as a 
fluorescent indicator, and mapped $\mathrm{O}_{2}$ evolution at ruthenium active sites of a composite electrode. ${ }^{18}$

More recently, fluorescence modulation of electrochemical reactions was followed at closed bipolar electrodes where the redox reaction of interest was coupled to a fluorogenic reaction. Imaging the fluorescence at one pole of the bipolar electrode gave information about the electrochemical reaction occurring at the other pole. ${ }^{19-22}$ In the same philosophy, a recent work reported that fluorescence modulation at the counter electrode could afford a quantitative characterization of the reaction occurring at the working electrode. ${ }^{23}$ The electrode surface where the reaction of interest occurs was also directly imaged either via direct or indirect fluorescence modulation. ${ }^{24}$

${ }^{25}$ Bohn et al. recorded the fluorescence arising from single-ET events of immobilized flavin adenine dinucleotide (FAD) or flavin mononucleotide freely diffusing across an array of nanopores enabling thus single molecule occupancy and confinement of the optical radiation. ${ }^{26-28}$

Fluorescence microscopy can provide crucial data about the interface reactivity since it enables monitoring chemical concentration gradients at electrochemical interfaces. One possibility is to image the plane perpendicular to the electrode surface. $^{29-31}$ Another possibility is to rebuild 3D profiles thanks to fluorescence confocal laser scanning microscopy (FCLSM). In FCLSM, the photons emitted are collected from an optical "slice" that corresponds to a thin volume with a narrow field depth at a given focal plane. As a result, blurring usually due to out-of-focus light collection is significantly reduced. FCLSM offers also a unique way of scanning the volume in the axial $(\mathrm{z})$ direction to rebuild a $3 \mathrm{D}$ view from a series of $2 \mathrm{D}$ (xy) images. Unwin and coworkers used this technique in situ during electrochemical experiments to image $\mathrm{pH}$ gradients induced by proton-coupled electrochemical reactions occurring at ultramicroelectrodes (UMEs) or microelectrode arrays (MEAs). The method proved to be valuable to decipher reaction mechanisms, and to detect defects in MEAs. More recently, the use of electro-fluorogenic systems proved that 3D fluorescence imaging could be a priori applied to any electrochemical reaction that involves the production or consumption of fluorescent species. ${ }^{32-34}$

To the best of our knowledge, recording FCLSM under electrochemical potential control has not been applied to the case of enzymatic catalysis. It might be surprising as microscopy is a very well established method in biology and is an intuitive way to get spatially resolved information. Here, we report for the first time such methodology coupling to characterize $\mathrm{O}_{2}$ reduction by the multi copper oxidase bilirubin oxidase (BOD) from the mesophilic fungus Myrothecium verrucaria
$(M v \mathrm{BOD})$. This enzyme catalyzes the 4 electron-4 proton reduction of $\mathrm{O}_{2}$ into water thanks to an active site composed of 4 copper nuclei. ${ }^{35}$ Current under catalytic turnover and fluorescence evolution at the electrode surface were recorded simultaneously in the presence of fluorescein. We demonstrate first how to reconcile specific constraints linked to both enzyme electrochemistry (like the need for adequate electrolyte and $\mathrm{pH}$, or the absence of inhibitors) and fluorescence microscopy (which relies on photon-emitting species). $M v \mathrm{BOD}$ is then immobilized at a carbon electrode that directly provides the electrons necessary for the reaction under electrochemical potential control. ET is coupled to proton transfer, so that a local $\mathrm{pH}$ change is a direct consequence of enzyme activity. This $\mathrm{pH}$ change in turn modulates the fluorescence properties of an adequate $\mathrm{pH}$-sensitive fluorophore (scheme 1). We show that this strategy enables imaging indirectly enzymatic catalysis by recording fluorescence evolution at the electrode surface during classical electrochemical experiments. We discuss the potentiality of the method to characterize spatial heterogeneity in electro-enzymatic catalysis. We especially highlight that the coupling method allows characterizing changes in enzyme environment upon operation, with the specific example of local basification expected to influence long-term biocatalyis.

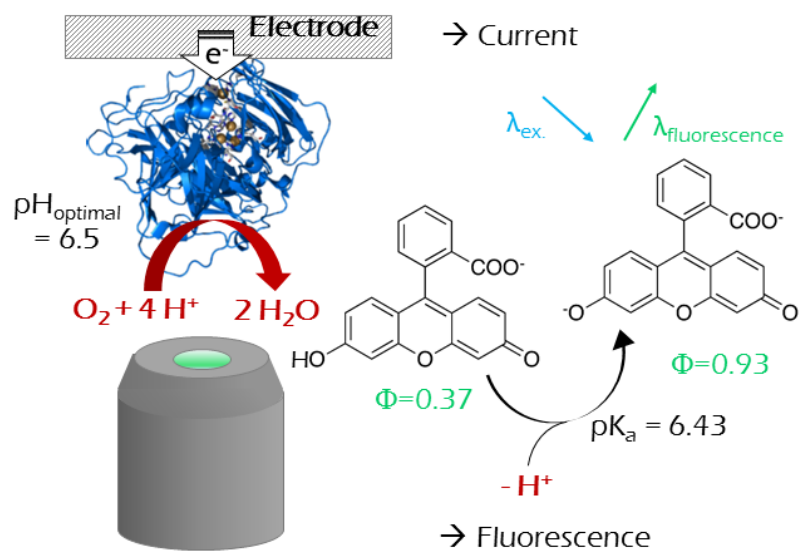

Scheme 1- Illustration of the experimental strategy. The working electrode provides the electrons necessary for the enzymatic $\mathrm{O}_{2}$-reduction. Simultaneously, protons consumption changes the $\mathrm{pH}$ locally, which in turn modulates the fluorescence of fluorescein whose quantum yield $(\Phi)$ is $\mathrm{pH}$-dependent. The change of fluorescence intensity is recorded through the objective by fluorescence confocal laser scanning microscopy (FCLSM).

\section{EXPERIMENTAL SECTION}


Bioelectrochemistry. The custom-made carbon electrode is a graphite $\operatorname{rod}(\varnothing=2.5 \mathrm{~mm})$ connected with a thin metal wire and inserted in a rubber sheath for electrical insulation. In this configuration only a disc is exposed to the enzyme solution (during electrode modification) and to the electrolyte (during electrochemical experiments). Enzyme (bilirubin oxidase from Myrothecium verrucaria, Amano, Japan) is adsorbed on the graphite by dipping the electrode in a $10 \mu \mathrm{M}$ enzyme solution in $50 \mathrm{mM}$ phosphate buffer $\mathrm{pH} 6$ for 10 minutes at $4^{\circ} \mathrm{C}$. The electrode used in figure 1 and 2 is a custom-made GC electrode modified by electrochemical oxidation of 4aminobenzoic acid, following a procedure described previous$\mathrm{ly}^{36}$, and thus bearing benzoic acid groups. Enzyme is adsorbed on the carbon disc following the same procedure than that used for GC electrode. The bioelectrochemical experiments are realized in a conventional 3-electrode cell: the reference electrode is a $\mathrm{Ag} / \mathrm{AgCl}$ reference electrode (Ametek) separated from the electrolyte by a $\mathrm{NaCl}$ junction, and the counter electrode is a platinum wire. Temperature is regulated at $25^{\circ} \mathrm{C}$ using a thermostated bath. The electrolyte $(50 \mathrm{mM} \mathrm{pH}$ 6 phosphate buffer, unless otherwise specified) is saturated with $\mathrm{O}_{2}$ prior to the experiment, and $\mathrm{O}_{2}$-flux is maintained over the electrolyte during all the duration of the experiment. Error bars are calculated based on at least three experiments.

In situ fluorescence microscopy during electrochemical experiments. The 3-electrode custom-made optoelectrochemical cell equipped with a $170 \mu \mathrm{m}$-thick glass window is placed above the objective of an inverted fluorescence confocal microscope Olympus FV1000-IX81. Images were acquired using an Olympus 5X objective MPLFLN (NA 0.15) or an Olympus 20X objective MPLFLN (NA 0.40). The cell can be finely moved in the three directions of space thanks to a manual micromanipulator to enable precise positioning and focus. The laser beam passes through the objective, which faces the working electrode surface, and the emitted light is also collected through the objective. A scheme of the setup is shown in $\mathrm{ref}^{34}$. A precise focus on the electrode surface is obtained by maximizing light intensity in laser reflection. In that case, the laser wavelength is $\lambda=543 \mathrm{~nm}$ and light is collected in the wavelength range $500-580 \mathrm{~nm}$. The working electrode is separated from the bottom of the electrochemical cell by a distance of a few mm. Images are acquired in situ during voltammetric or chronoamperometric experiments performed in a solution containing fluorescein, with an initial $\mathrm{pH}$ of 5.4. Fluorescence is recorded from a given confocal volume centered at the electrode surface or within the solution at incremental distances from the electrode surface. The wavelength of excitation is $\lambda=488 \mathrm{~nm}$, and fluorescence is collected in the range 500-580 $\mathrm{nm}$ after filtering through a $488 / 543 / 633 \mathrm{~nm}$ dichroic mirror. A titanium wire is used as a counter electrode and a $\mathrm{Ag} / \mathrm{AgCl} / \mathrm{KCl}$ sat. electrode is used as reference. Experiments have been repeated at least three times.

\section{RESULTS AND DISCUSSION}

Enzymatic catalysis in unconventional conditions. MvBOD works at ambient temperature and its optimum activity is observed at $\mathrm{pH} 6.5 .^{37} \mathrm{We}$ selected as a $\mathrm{pH}$-dependent dye fluorescein whose fluorescence properties strongly depend on its protonation state. The absorption spectrum of the di-anionic form features a sharp band at $490 \mathrm{~nm}\left(\varepsilon=76900 \mathrm{~L} \mathrm{~mol}^{-1} \mathrm{~cm}^{-1}\right)$
A

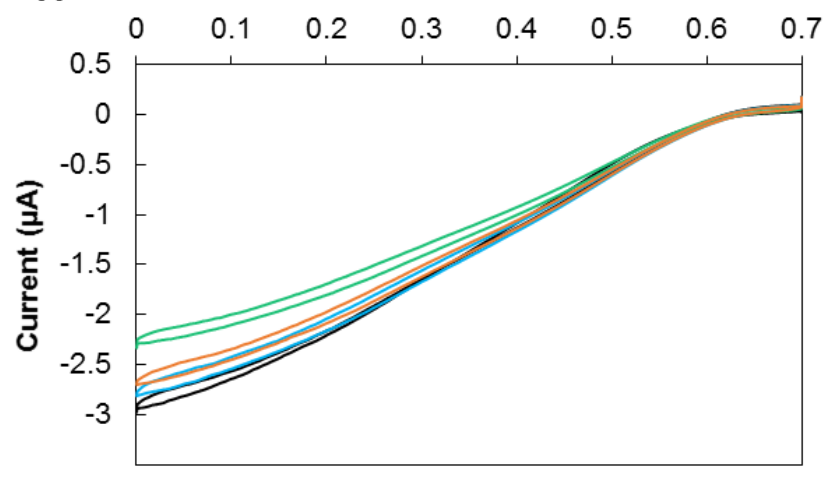

B Fluorescein concentration $(\mu \mathrm{M})$ : $\begin{array}{llllllll}10 & 20 & 50 & 75 & 100 & 150 & 200 & 200\end{array}$

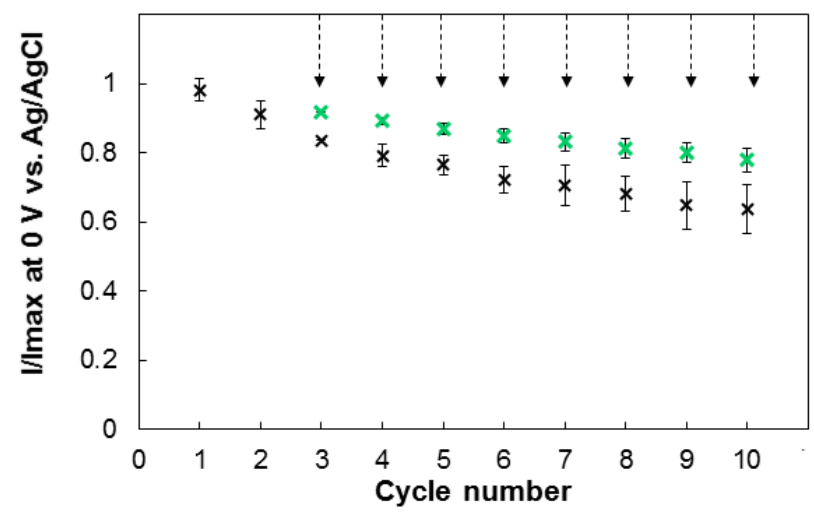

C Content of unbuffered electrolyte (\%) :

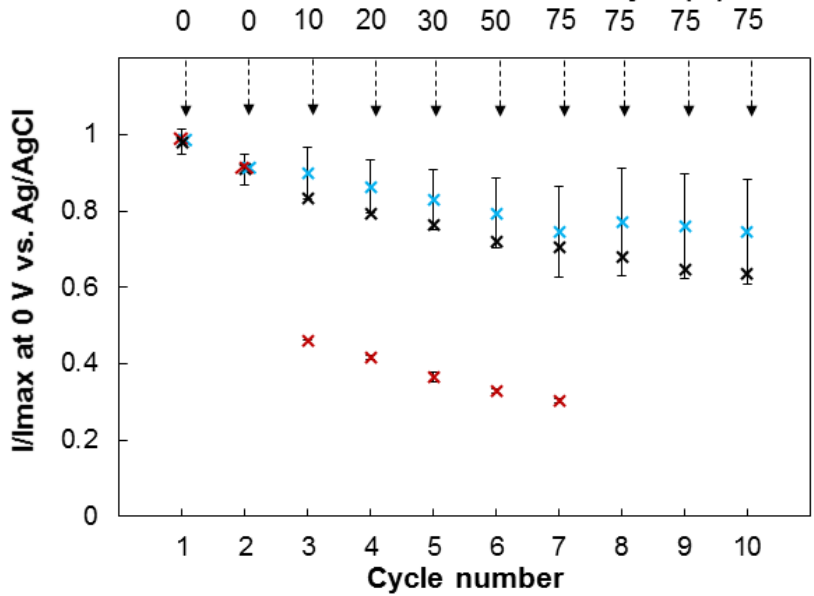

whereas the mono-anion absorption is blue-shifted and weaker with two bands of equal magnitude at 453 and $472 \mathrm{~nm}(\varepsilon=$ $\left.29000 \mathrm{~L} \mathrm{~mol}^{-1} \mathrm{~cm}^{-1}\right){ }^{38}$ On the other hand, the fluorescence maximum wavelength is the same at $525 \mathrm{~nm}$ with a similar lifetime (3-4 ns). However, the quantum yield is $\Phi=0.37$ for the mono-anionic form while it rises to $\Phi=0.93$ for the dianionic form. The corresponding $\mathrm{pK}_{\mathrm{a}}$ is 6.43 . Therefore, when the solution initial $\mathrm{pH}$ is lower than this $\mathrm{pK}_{\mathrm{a}}, \mathrm{a} \mathrm{pH}$ increase does result in a fluorescence enhancement. In our case, the optimum $\mathrm{pH}$ of the enzyme ( $\mathrm{pH}$ 6.5) perfectly matches the $\mathrm{pK}_{\mathrm{a}}$ of the fluorophore, so that it enables enzyme operation in conditions close to its optimum. However, multi-copper oxidases (among which BOD) are known to be inhibited by several anions. Therefore, a prerequisite is to ensure that the en- 
zyme activity does not suffer from the presence of fluorescein, which can exhibit both its mono- and di-anionic forms around $\mathrm{pH}$ 6. Moreover, the fluorescence evolution at the electrode surface should be exclusively related to $\mathrm{pH}$ changes. Fluorescein is a priori not electroactive in the considered potential range, but undergoes an irreversible oxidation at higher potentials. ${ }^{39}$ We therefore compared the electrocatalytic behavior of the enzyme in the absence or presence of the fluorophore (Figure 1). A typical cyclic voltammogram (CV) of enzymatic $\mathrm{O}_{2}$ reduction at a $\mathrm{GC}$ electrode is displayed in Figure 1A. Incremental addition of fluorescein from 10 to $200 \mu \mathrm{M}$ does not affect the shape of the signal, and no additional electrochemical signal is observed from 0 to $0.7 \mathrm{~V}$ vs. $\mathrm{Ag} / \mathrm{AgCl}$. We also studied the electrochemical behavior of fluorescein over an extended potential range (Figure S1), which further confirmed its electro-inactivity in the concerned potential window although an irreversible oxidation occurs at slightly higher potentials. Moreover, the evolution of the current intensity upon cycling is comparable in the absence or presence of fluorescein, showing only a slight decrease over time in both cases (Figure 1B). This decrease over cycles is quasi systematically observed with enzyme electrodes and has long been referred to as "film loss". However, recent studies have shown that it mostly linked to other processes than enzyme leakage from the electrode surface, the applied potential and local electric field playing a major role on the enzyme conformation. 3, 6, 40 From this initial assessment, we conclude that fluorescein is electro-inactive in the studied potential window, and that it does neither inhibit nor destabilize significantly the immobilized enzyme, even at concentrations 10 times higher than required to visualize $\mathrm{pH}$ evolution without ambiguity.

Figure 1. Enzymatic catalysis in unconventional conditions. A and B: Influence of fluorescein on the catalytic response. C: Selection of an appropriate electrolyte. A: Evolution of $\mathrm{O}_{2}$ enzymatic reduction wave without fluorescein $\left(1^{\text {st }}\right.$ cycle: black, $2^{\text {nd }}$ cycle: blue) then upon incremental addition of fluorescein $\left(3^{\text {rd }}\right.$ cycle: $10 \mu \mathrm{M}$ (orange) and $10^{\text {th }}$ cycle: $200 \mu \mathrm{M}$ (green). B: Evolution of normalized current intensity at $0 \mathrm{~V}$ vs. $\mathrm{Ag} / \mathrm{AgCl}$ during successive cycles without fluorescein (black) and upon incremental addition of fluorescein (green). The arrows indicate the fluorescein concentrations (in $\mu \mathrm{M}$ ). C: Evolution of normalized current intensity of $\mathrm{O}_{2}$ enzymatic reduction at $0 \mathrm{~V}$ vs. $\mathrm{Ag} / \mathrm{AgCl}$ during successive cycles in $100 \mathrm{mM}$ phosphate buffer (black), or in $100 \mathrm{mM}$ phosphate buffer with increasing proportions of ammonium sulfate (blue) or sodium perchlorate (red). The arrows indicate the increasing percentages of unbuffered electrolyte. The overall electrolyte concentration is kept constant. Experimental conditions: $M v \mathrm{BOD}$ immobilized at a GC electrode, Electrolyte $=100 \mathrm{mM}$ phosphate buffer $(\mathrm{A}$ and $\mathrm{B})$, or $100 \mathrm{mM}$ phosphate buffer with increasing proportions of ammonium sulfate or sodium perchlorate (C), $\mathrm{pH} 6, \mathrm{R} . \mathrm{T} ., v=5$ $\mathrm{mV} \mathrm{s}^{-1}$, quiescent electrolyte under $\mathrm{O}_{2}$ atmosphere.

Due to the strong pH dependency of enzyme activity, enzyme electrochemistry is usually recorded in buffered electrolytes with typical molarities in the range 50-200 mM. Practically, it means that any $\mathrm{pH}$ change provoked by the enzymatic reaction at the electrochemical interface should be counter-balanced by a bulk reaction with the buffer weak acid/base, thus ensuring a constant $\mathrm{pH}$ in the enzyme environment. Since our imaging strategy relies on this $\mathrm{pH}$ change, the use of high molarity buffer as an electrolyte was a priori excluded. Therefore, we looked for a convenient non-buffered electrolyte, keeping in mind once more that $M v \mathrm{BOD}$ is sensitive to several anions. It is especially inhibited by halides, ${ }^{35}$ which excludes also the use of classic electrolytes such as $\mathrm{NaCl}, \mathrm{KCl}$ etc.

Figure $1 \mathrm{C}$ compares the evolution of the enzymatic $\mathrm{O}_{2}$ reduction current recorded during consecutive cycles performed either in phosphate buffer alone, or in phosphate buffer with an increasing concentration of two non-buffered electrolytes: ammonium sulfate and sodium perchlorate, respectively. The shape of the CVs is not affected by the presence of either sulfate or perchlorate anions (Figure S2). However, an immediate drop in current intensity is readily observed with only $10 \%$ sodium perchlorate in the buffer solution. Such a drop cannot be attributed to a $\mathrm{pH}$ modification since it is observed even for small volume addition, for which no significant $\mathrm{pH}$ change occurs. Electrolyte concentration is also kept constant, so that the effect of ionic strength can be ruled out. Sodium perchlorate is more likely an inhibitor of the enzyme activity. On the contrary, no significant change is observed upon addition of ammonium sulfate, even for the highest concentrations tested, i.e. for the most diluted buffer. In this condition (75/25 un-buffered/buffered electrolyte), the expected local basification at the interface is not sufficient to affect significantly the enzyme activity over the time lapse of the experiment (here 1 cycle corresponds to $280 \mathrm{~s}$ ). The reasonable stability observed over 10 cycles in $100 \%$ ammonium sulfate (Figure S3) justifies its use for further experiments.

Fluorescence evolution at the electrode surface during the enzymatic reaction. After the initial electrochemical assessment, we recorded in situ microscopy by using a homemade opto-electrochemical cell with a transparent optical window (see experimental section in the SI). We recorded the variation of fluorescence during a cyclic voltammetry experiment with the focal plane set at the electrode surface $(z=0)$. In the absence of labelling and since the laser wavelength does not correspond to tryptophan excitation $(295 \mathrm{~nm})$, the protein structure does not emit any fluorescence. Figure 2 shows a series of images collected at regular potential intervals with an acquisition time of $2.6 \mathrm{~s}$ per image. The initial electrolyte $\mathrm{pH}$ was set at 5.4 , i.e. one $\mathrm{pH}$ unit below the fluorescein $\mathrm{pK}_{\mathrm{a}}$, which means that the mono-anionic form is predominant at the beginning of the experiment. The electrode is first equilibrated in the absence of enzymatic catalysis by applying a potential of $0.7 \mathrm{~V}$ vs. $\mathrm{Ag} / \mathrm{AgCl}$. The "background" fluorescence signal at this potential is solely due to the contribution from the fluorescein mono-anionic form. When the voltage is swept at a constant scan rate towards more reducing potentials, fluorescence patches appear at the electrode surface (disk diameter: $2.5 \mathrm{~mm}$ ) and spread over the whole electroactive surface. On the contrary, the fluorescence does not change in the part corresponding to the insulating shell surrounding the electroactive surface area. This behavior emphasizes that $\mathrm{O}_{2}-$ reduction does solely occur in the active part of the electrode surface that can provide electrons to the immobilized enzyme. The fluorescence intensity is maximal at $0 \mathrm{~V}$ vs. $\mathrm{Ag} / \mathrm{AgCl}$ (in the observed potential range), which is also self-consistent with a maximal current density. In the reverse scan, when the potential is swept back towards positive values, the fluores- 
cence decreases until a level close to the initial one. As a final proof that the fluorescence is related to the enzyme reaction, we also show a negative control experiment performed without the enzyme in Figure S4.

Figure 2B shows the evolution of the current and fluorescence recorded simultaneously at an electrode during a similar $\mathrm{CV}$, in the presence and absence of enzyme. The fluorescence was averaged over the entire electrode surface for each frame of the corresponding video. When comparing electrochemical and fluorescence signals, it is obvious that the fluorescence channel is much more sensitive than the electrochemical one. This is expected as the transfer of a single electron promotes the emission of numerous photons, and the photo-detection is more sensitive than the electrochemical detection. Although the fluorescence evolution recorded during a $\mathrm{CV}$ performed in the absence of enzyme is not null, it is dramatically lower than the one recorded in the presence of enzyme. This is a clear proof that we monitored indeed the signature of enzymatic $\mathrm{O}_{2}$ reduction. An important conclusion is that the enzyme electroactivity is maintained upon exposition to laser radiation, ensuring the validity of the method for enzymatic studies. The slight slope visible in electrochemistry in the absence of enzyme can result from a resistive behavior of the electrode, or a small abiotic reduction current. The latter could be related to a modification of the interfacial $\mathrm{pH}$ and cause the noise observed in fluorescence measurement. Considering this, we always measured a blank signal before the enzymatic measurements and considered only the differential signal with respect to this blank. Moreover, we studied the influence of fluorophore concentration on the fluorescence resulting both from the enzyme activity (the "signal") and the abiotic phenomena (the "noise") (Figure S5). We therefore set fluorescein concentration to $10 \mu \mathrm{M}$, which gives the most reliable $\mathrm{S} / \mathrm{N}$ ratio. Interestingly, fluorescence spectra recorded for different fluorophore concentrations also indicate that this concentration leads to the maximal emission (Figure S6), due to the innerfilter effect or self-quenching at higher concentrations.

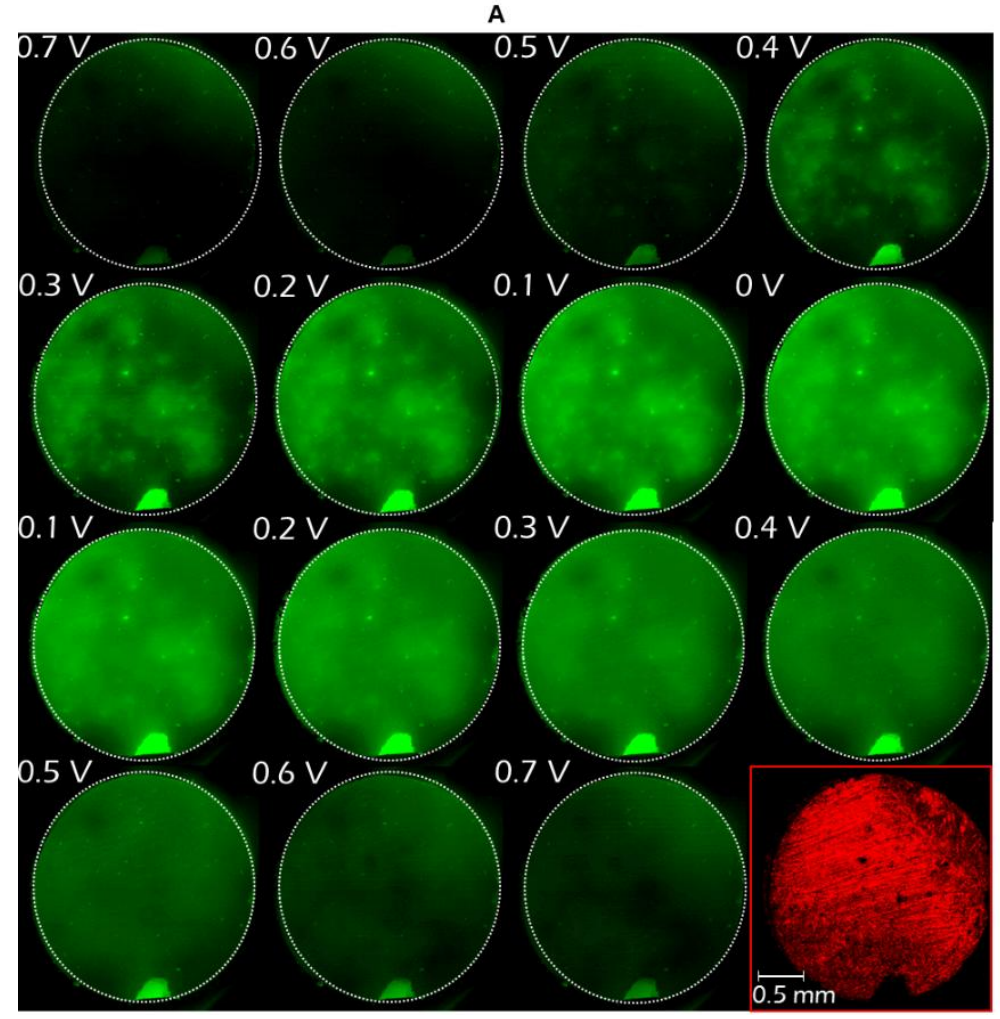

B

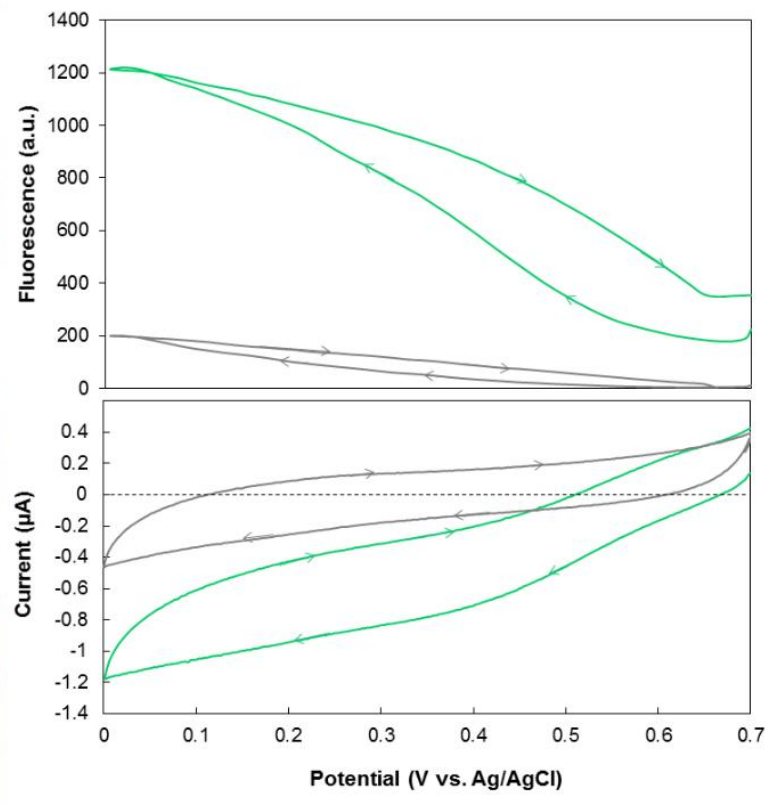

Figure 2. Fluorescence evolution at the electrode surface during the enzymatic reaction. Panel A: Images extracted at different potentials from a video recorded during a cyclic voltammetry of $\mathrm{O}_{2}$ reduction by the immobilized BOD from Myrothecium verrucaria. To enable better localization, the electrode disk is surrounded with a white dotted circle. $\mathrm{E}_{\text {initial }}=0.7 \mathrm{~V}, \mathrm{E}_{\mathrm{vertex}}=0 \mathrm{~V}, v=5 \mathrm{mV} \mathrm{s}{ }^{-1}$, and the indicated potentials are quoted against the $\mathrm{Ag} / \mathrm{AgCl}$ reference electrode. The bottom right image in red shows the same electrode surface imaged by laser reflection at $\lambda=543 \mathrm{~nm}$. Panel B: Electro-fluorogram (Fluorescence vs. Potential, top) and Voltamperogram (Current vs. Potential, bottom) recorded simultaneously at the electrode during cyclic voltammetry with the enzyme (green curves) and without the enzyme (grey curves). Experimental conditions: $M v$ BOD immobilized at a graphite electrode, electrolyte $=100 \mathrm{mM}$ ammonium sulfate, 10 $\mu \mathrm{M}$ fluorescein, initial $\mathrm{pH}=5.4, \mathrm{R} . \mathrm{T}$., quiescent electrolyte under air atmosphere. Wavelength of the excitation laser: $\lambda_{\text {excitation }}=488 \mathrm{~nm}$.

The potential-dependency of fluorescence at the electrode surface and current intensity follow a similar trend (Figure 2B). $\mathrm{O}_{2}$ reduction current appears when the potential is scanned towards reducing values, which corresponds to increasing overpotentials while fluorescence does simultaneous- ly increase. Both current and fluorescence maxima are reached at $\mathrm{E}=0 \mathrm{~V}$ vs. $\mathrm{Ag} / \mathrm{AgCl}$. A consistent behavior is observed on the reverse scan with a diminution of both current and fluorescence with decreasing overpotentials. However, a closer look at the data underlines that they are not strictly proportional. 
First, a small delay can be observed, i.e. the fluorescence starts to increase slightly after the current. This is not surprising as the image acquisition in FCLSM relies on a pixel-by-pixel sample scan. Therefore, the potential slightly varies between the beginning and the end of a single image construction. Moreover, the correlation diagram (Figure S7) shows a monotonous but nonlinear relationship. In a recent work, epifluorescence microscopy was used to follow fluorescence evolution of a fluorogenic reporter at the counter electrode and relate it to the current linked to the reaction of interest occurring at the working electrode. The authors reported that fluorescence and current did not match when using a millimetric electrode. $^{23}$ Previous studies by Ackerman et al. ${ }^{41}$ and by Zhang et al. ${ }^{20}$ showed that when using wide field microscopy the time derivative of fluorescence was linearly correlated with the current flowing through the electrode surface. This is expected because the fluorescence recorded in that case results from the contributions of the total number of fluorescent molecules. According to Faraday's law, since these latter are formed exclusively by the electrochemical reaction, their amount and therefore the fluorescence are also proportional to the total charge transferred during the electrochemical experiment.

In the present study, a key factor is the slow scan rate that enables steady state of the enzyme reaction to be reached at every potential. Moreover, the measurements were performed with a confocal microscope. In FCLSM, a pinhole is positioned in front of the detector through the optical pathway, thus preventing out-of-focus light from reaching the detector. This means that fluorescence evolution is recorded in a thin volume centered on the focal plane, contrarily to wide field microscopy where fluorescence is recorded in the whole electrolyte volume. A direct consequence is that the corresponding fluorescence signal is tuned by several in- and outflows (reaction, mass transport to and from the electrochemical interface). The evolution of fluorescence intensity should therefore be directly correlated with the rate of the electrochemical reaction, and thus to the current intensity. In the present case, the thickness of the probed volume is in the range of $100 \mu \mathrm{m}$. This is due to the low magnification and numerical aperture of the objective employed, which was chosen on purpose to enable observation of the whole macro-electrode surface. However, this dimension remains smaller than the expected extension of the diffusion layer on the timescale of the experiment (between 0.3 and $0.5 \mathrm{~mm}$ for a macroelectrode at steady state in the absence of electrolyte agitation). The goal of this study was to demonstrate the feasibility of this approach. It is obvious that the use of microelectrodes in combination with high magnification objectives with large numerical aperture will be necessary to allow precise quantification of the results in further studies.
A last striking detail is the presence of a hysteresis on both signals. It is easily interpreted in the electrochemical signal as a capacitive current proportional to the product of the electrode surface and the capacitance of the electrochemical double layer. However, the hysteresis is inverted in the fluorescence channel with the intensity observed during the reverse scan being above that recorded during the forward scan. Although more intriguing, it is consistent with the small time delay separating the two signals on the forward scan. A further explanation might be a mechanistic difference in the signal generation, since fluorescence is switched on when the electrode potential is swept towards $0 \mathrm{~V}$ and switched off in the opposite sweep.

Mapping heterogeneity by FCLSM. In the enzymatic experiment, several bright fluorescence spots with tens-ofmicrometers sizes can be noticed on the electrode surface. For comparison, the red image at the bottom right in Figure 2 shows the topography of the electrode surface as observed by laser reflection. The superimposition of enzymatic and topography images (Figure S8) reveals an excellent agreement between these bright spots and the holes observed on the topographic image. This is understandable since defects (induced by sonication treatment, see Figure S9) cause local changes of the apparent thickness of the confocal volume that is probed when focalizing on the electrode plane. A great advantage of FCLSM compared to wide field microscopy is also the possibility to rebuild three-dimensional images. For this purpose, fluorescence is recorded at different focal planes by moving vertically either the objective or the sample. The $3 \mathrm{D}$ image is then reconstructed by stacking the series of images recorded at different $\mathrm{z}$ positions, which can be considered as optical slices. In the case of a coupling with electrochemistry, it enables rebuilding the diffusion layer of an electroactive fluorophore or a fluorescent reporter, or exploring cavities at the electrode surface (or in the electrode volume). In figure 3, Panel A, we show the fluorescence intensity during enzymatic reaction at an electrode similar to the one that was previously characterized (A1), and the corresponding 3D image (A2). This electrode exhibits a heterogeneous fluorescence with small bright dots distributed in the disk and around it. These latter appear as sharp peaks inside the tomographic $3 \mathrm{D}$ reconstruction and can be attributed to the presence of micro-cavities. Moreover, their depth can be estimated to $\sim 20 \mu \mathrm{m}$ by FCLSM using an objective with both higher magnification and higher numerical aperture (A3). Enzymes could accumulate in these cavities, and cause a higher local reactivity. For comparison, we show a second electrode obtained after the same polishing but omitting the sonication step (Panel B), which "erases" the heterogeneity. In that case, fluorescence distribution over the electrode surface is homogeneous and a rather smooth limit is observed on the 3D image at the electrolyte/electrode interface. 
A

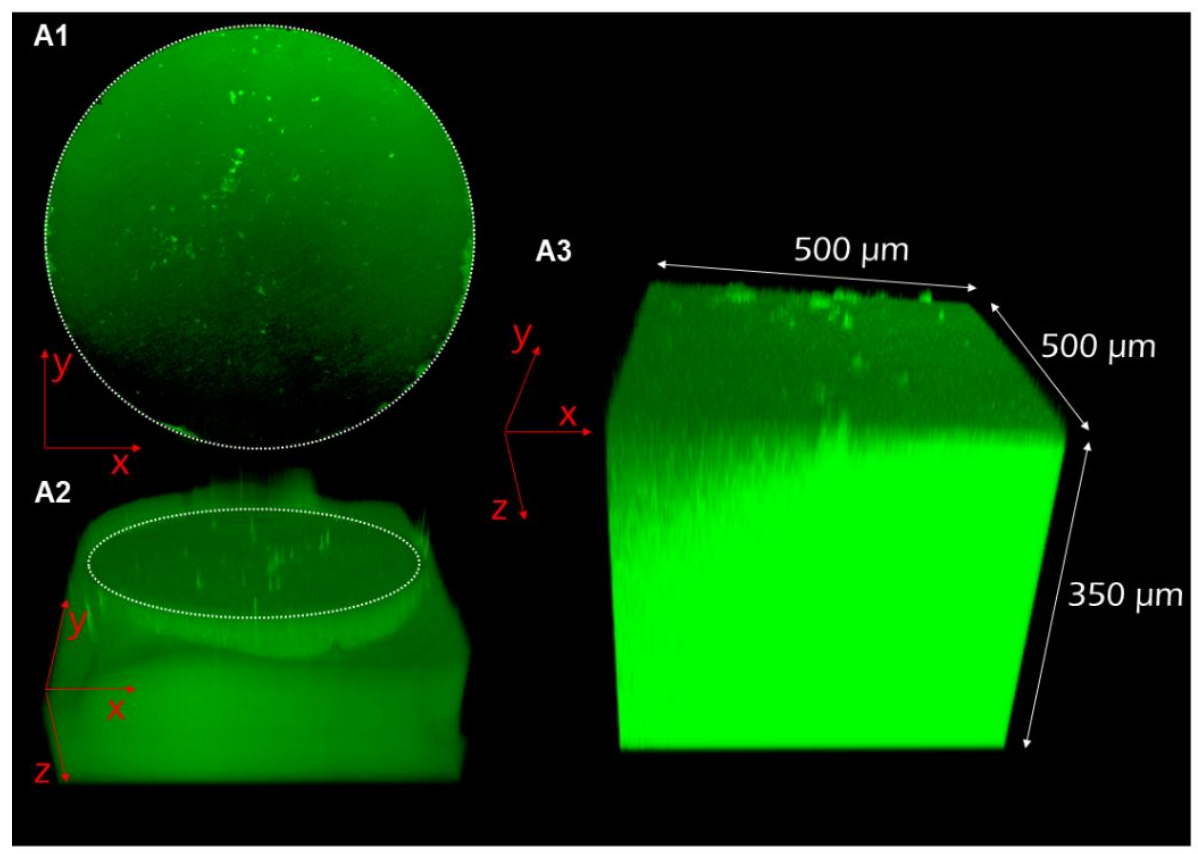

B

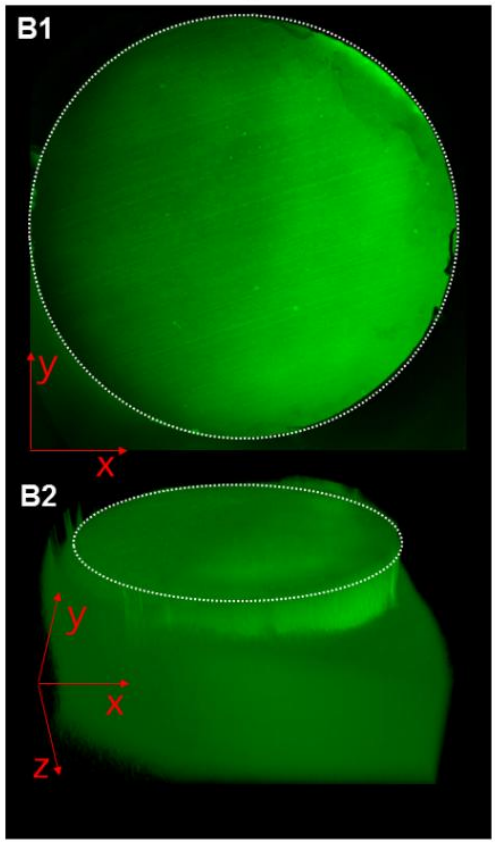

Figure 3. Microstructure and reactivity of the graphite electrode examined by FCLSM. Panel A: Fluorescence during $\mathrm{O}_{2}$-reduction at an enzymatic electrode prepared as described in the experimental section. To enable better localization, the electrode disk is surrounded with a white dotted circle (diameter $2.5 \mathrm{~mm}$ ). A1: image extracted from cyclic voltammetry; A2 and A3: 3D images realized by z-stacking at a constant potential $\mathrm{E}=0.3 \mathrm{~V}$ vs. $\mathrm{Ag} / \mathrm{AgCl}$ at two different magnifications. The objective magnifications are $5 \mathrm{X}$ and $20 \mathrm{X}$ for $\mathbf{A 2}$ and $\mathbf{A 3}$ respectively, and the corresponding numerical apertures are 0.15 and 0.45 . For comparison, $\mathrm{O}_{2}$-reduction at an enzymatic polished but notsonicated electrode surface is illustrated in Panel B. B1: image extracted from cyclic voltammetry; B2: 3D images realized by z-stacking at a constant potential $\mathrm{E}=0.3 \mathrm{~V}$ vs. $\mathrm{Ag} / \mathrm{AgCl}$ at the lowest magnification (Characteristics of the objective: mag.= 5X, N.A.= 0.15). Experi-

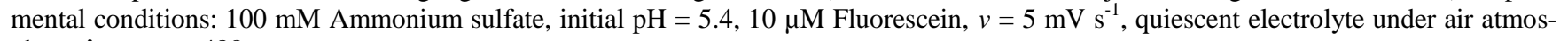
phere. $\lambda_{\text {excitation }}=488 \mathrm{~nm}$.

\section{Enzyme-driven $\mathrm{pH}$ modulation despite the presence of buff-} $\boldsymbol{e r}$. We considered a priori that an un-buffered electrolyte is more appropriate to image $\mathrm{pH}$ modulations. However, the influence of buffer concentration on the fluorescence evolution is highly relevant to improve the understanding of enzymatic catalysis. First, it can provide evidence if the presence of buffer is sufficient to counter-balance $\mathrm{pH}$ changes occurring at the bioelectrode. Second, an adequate buffer concentration should limit the expansion of the proton diffusion layer, enabling fluorescence changes only at the exact localization of proton consumption, and thus facilitating the mapping of electrocatalysis. A first experiment was performed in chronoamperometry mode. The electrode potential was held at $0.3 \mathrm{~V}$ vs. $\mathrm{Ag} / \mathrm{AgCl}$, i.e. in the potential range where enzymatic catalysis occurs readily while the abiotic fluorescence gives a reasonable noise. Theoretically, the expansion of the diffusion layer is expected to be time-dependent in the absence of forced convection. However, in chronoamperometry a steady state current is reached quite fast, which indicates that other phenomena than diffusion are involved in mass transport of $\mathrm{O}_{2}$ and $\mathrm{H}^{+}$(else current would gradually decrease due to the constant expansion of the diffusion layer) or that the current at the electrode is not limited by the mass transport of the substrate. Fluorescence is recorded after reaching this steady state current (typically about $1 \mu \mathrm{A}$ ), which is similar whatever the buffer content. The stability of enzyme is sufficient on the timescale of the experiment $(<5$ minutes) and current remains constant. Fluorescence is recorded in planes parallel to the electrode disk at incremental distances from the surface. 3D images are then re-constructed by stacking all these "optical slices". Figure 4A presents radial cross-sections of these 3D images for four different buffer contents: 0, 10, 25 and 50\% $\mathrm{V}: \mathrm{V}$ of phosphate-citrate buffer in $100 \mathrm{mM}$ ammonium sulfate. The repartition of fluorescence and therefore of protons in the volume of the electrolyte solution in the vicinity of the electrode surface can be visualized, revealing the proton gradient direction from the electrode surface towards the bulk electrolyte. As expected, the expansion of the diffusion layer drastically decreases with increasing buffer concentrations, ${ }^{29}$ being almost undetectable at concentrations higher than 50 $\mathrm{mM}$ buffer. The shape and dimensions of the diffusion layer can also be characterized. The asymmetric shape of the diffusion layer oriented towards the upper right corner was already observed $^{33}$ and was ascribed to the migration of charged species towards the counter electrode. The electrode surface is separated from the glass bottom by a few $\mathrm{mm}$, so that any restriction of the diffusion can be ruled out. It is visible here that holes indeed lead in some cases to higher local $\mathrm{pH}$ change in the diffusion layer (indicated for instance by the arrows in the second or third picture). Except of this, catalysis seems rather homogeneously distributed over the electrode surface. 


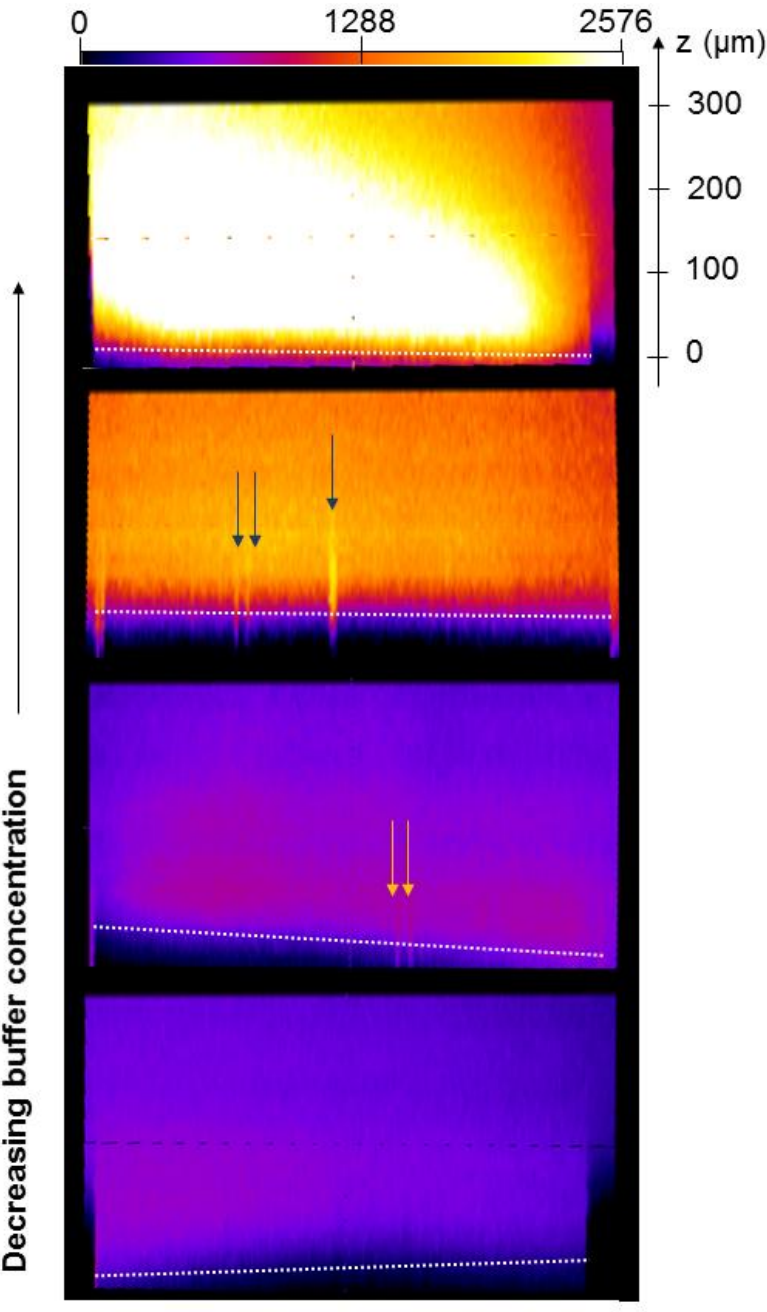

B

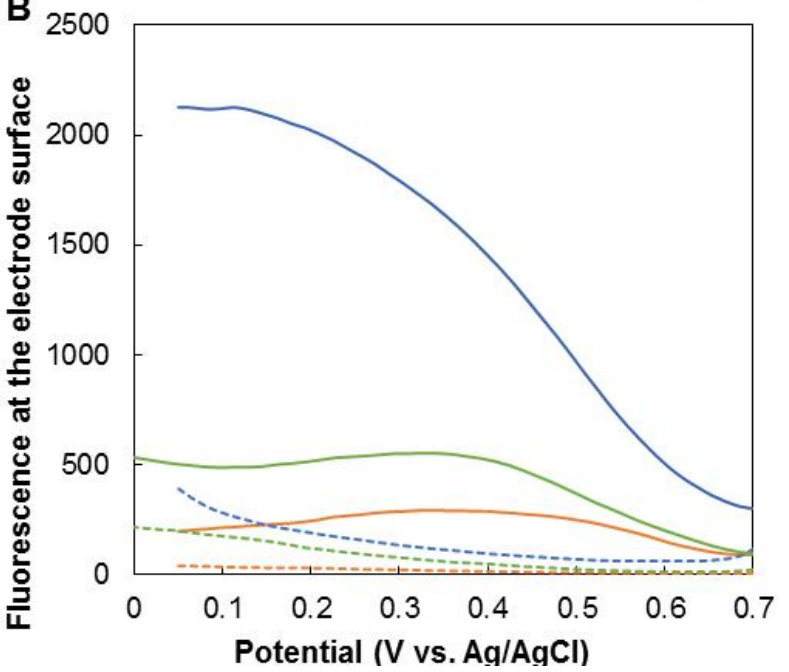

Figure 4. Enzyme-driven pH modulation despite the presence of buffer. A: Profile views of the proton diffusion layer at the bioelectrode during a chronoamperometry experiment for different proportions of phosphate-citrate buffer in $100 \mathrm{mM}$ ammonium sulfate: $0 \% \mathrm{~V}: \mathrm{V}$ (top); $10 \% \mathrm{~V}: \mathrm{V}$ (second); $25 \% \mathrm{~V}: \mathrm{V}$ (third) and $50 \% \mathrm{~V}: \mathrm{V}$ (bottom). The dotted line indicates the electrode surface. The arrows indicate the presence of holes on the electrode surface. Electrode potential is held at $0.3 \mathrm{~V}$ vs. $\mathrm{Ag} / \mathrm{AgCl}$ and current is about $1 \mu \mathrm{A}$ in all cases. Initial $\mathrm{pH}=5.4$, [Fluorescein] $=$ $10 \mu \mathrm{M}$, quiescent electrolyte under air atmosphere. $\lambda_{\text {excitation }}=488$ $\mathrm{nm}$. The scale bar at the top indicates the fluorescence intensity scale. B: Fluorescence evolution at the electrode surface during linear scan voltammetry of $\mathrm{O}_{2}$ reduction for different proportions of phosphate-citrate buffer in $100 \mathrm{mM}$ ammonium sulfate: $0 \%$ $\mathrm{V}: \mathrm{V}$ (blue lines); $25 \% \mathrm{~V}: \mathrm{V}$ (green lines) and $50 \% \mathrm{~V}: \mathrm{V}$ (orange lines). The experiment is performed in the absence (dotted lines) or presence of enzyme (solid lines).

The electro-fluorogram presented in Figure 4B is recorded with the focal plane set at the electrode surface. It compares fluorescence evolution in the absence or presence of $50 \mathrm{mM}$ phosphate-citrate buffer. The initial $\mathrm{pH}$ is the same. Although fluorescence increase with decreasing potentials is much lower in the presence of buffer, the $\mathrm{pH}$ change can still be clearly evidenced in that latter case. This means that enzymes immobilized at the electrode surface are indeed submitted to such a $\mathrm{pH}$ evolution, which can potentially affect their long-term activity or even damage their structure. This could be a reasonable explanation of destabilization classically encountered over cycling during voltammetry experiments. It was also shown previously that changing the electrolyte $\mathrm{pH}$ affects the rate of ET between the enzyme and the electrode, due to protein mobility on the electrode surface when the enzyme immobilization relies on electrostatic interactions. ${ }^{5}$ Our results thus suggest that the ET efficiency could actually be modulated during the course of the experiment. Moreover, this work underlines that a higher local activity (due to enzyme accumulation, or higher specific activity) potentially leads to a faster destabilization or an activity decrease, due to harsher submission to $\mathrm{pH}$ evolution.

\section{CONCLUSION}

This work provides a proof-of-concept visual characterization of the intrinsic reactivity of an enzymatic electrode thanks to confocal fluorescence microscopy implemented in situ during the electrochemical experiment. To the best of our knowledge, this is the first time that this kind of in situ coupling is used in the frame of enzyme catalysis at an electrode. The results described here show that the immobilized enzyme handles the focalized laser irradiation, and that this method gives access to spatially-resolved information inaccessible so far.

A fluorescence evolution directly correlated to enzymatic $\mathrm{O}_{2-}$ reduction at the electrode interface was observed, providing several requirements were fulfilled. Suitable pKa and electrochemical characteristics of the fluorophore, as well as a convenient electrolyte composition, are key prerequisites. We demonstrated that observing the electrode surface or the surrounding electrolyte by FCLSM during enzymatic electrocatalysis is a method of choice to get a fruitful additional level of information that cannot be obtained solely by conventional electrochemical techniques. In particular, such a coupled approach can be implemented to characterize the enzymatic electrode interface together with changes undergone during the enzymatic turnover. Key information extracted here are local changes in the enzyme environment at the electrode surface, and especially $\mathrm{pH}$ modulation even in the presence of buffered electrolyte.

The versatility of the method for investigating many different enzymes is ensured since most redox-active enzymes undergo proton-coupled electron transfers. In future studies, the use of microelectrodes in combination with high magnification objectives possessing large numerical aperture will allow precise quantification of the results and increase significantly the spatial resolution. Moreover, the use of a ratiometric dye 
might permit an exact quantification of the reaction rate and of the local $\mathrm{pH}$.

\section{ASSOCIATED CONTENT}

\section{Supporting Information}

Figure S1. Electrochemical reactivity of fluorescein; Figure S2. Selection of an appropriate electrolyte; Figure S3. Stability of the enzymatic signal in the ammonium sulfate electrolyte; Figure S4: Fluorescence evolution at the electrode surface in the absence of enzyme; Discussion around the Influence of Fluorophore concentration: delicate interplay between intensity and signal/noise ratio; Figure S5. Fluorescence evolution at the electrode surface during cyclic voltammetry for different concentrations of fluorescein; Figure S6. Spectra of fluorescein for different fluorescein concentrations; Figure S7. Correlation diagram of fluorescence and current intensity; Figure S8. Comparison of fluorescence localization during the enzymatic reaction and electrode surface microstructure; Figure S9. Microstructure of the graphite electrodes examined by scanning electron microscopy recorded ex situ

The Supporting Information is available free of charge on the ACS Publications website.

\section{AUTHOR INFORMATION}

\section{Corresponding Author}

* adepoulpiquet@imm.cnrs.fr

\section{Author Contributions}

All authors have given approval to the final version of the manuscript

\section{ACKNOWLEDGMENT}

The authors gratefully acknowledge CNRS Mission for Interdisciplinarity for the funding under the grant "Défi Imag'In MOMA", Frédérique Berger from Aix-Marseille University for the design and construction of the electrochemical cell enabling fluorescence microscopy measurements, and Amano Japan for kindly providing Myrothecium verrucaria Bilirubin oxidase. They also thank Dr. B. Goudeaux (ISM, Bordeaux, France) and Dr. Artemis Kosta (IMM, Marseille, France) for technical support, Pr. T. Doneux (ULB, Brussels, Belgium) and Dr. I. Mazurenko (BIP, Marseille, France) for fruitful discussions, and Dr. M.-T. GiudiciOrticoni (BIP, Marseille, France) for fruitful discussion and careful reading of the manuscript. H. M. Man acknowledges Région Sud and HySEAS for funding her $\mathrm{PhD}$ thesis.

\section{REFERENCES}

1. Ciaccafava, A.; Infossi, P.; Ilbert, M.; Guiral, M.; Lecomte, S.; Giudici-Orticoni, M. T.; Lojou, E., Electrochemistry, AFM, and PM-IRRA Spectroscopy of Immobilized Hydrogenase: Role of a Hydrophobic Helix in Enzyme Orientation for Efficient H-2 Oxidation. Angew. Chem.Int. Edit. 2012, 51 (4), 953-956.

2. Hitaishi, V. P.; Clement, R.; Bourassin, N.; Baaden, M.; de Poulpiquet, A.; Sacquin-Mora, S.; Ciaccafava, A.; Lojou, E., Controlling Redox Enzyme Orientation at Planar Electrodes. Catalysts 2018, 8 (5).

3. McArdle, T.; McNamara, T. P.; Fei, F.; Singh, K.; Blanford, C. F., Optimizing the Mass-Specific Activity of Bilirubin Oxidase Adlayers through Combined Electrochemical Quartz Crystal Microbalance and Dual Polarization
Interferometry Analyses. Acs Applied Materials \& Interfaces 2015, 7 (45), 25270-25280.

4. Gutierrez-Sanchez, C.; Ciaccafava, A.; Blanchard, P. Y.; Monsalve, K.; Giudici-Orticoni, M. T.; Lecomte, S.; Lojou, E., Efficiency of Enzymatic O-2 Reduction by Myrothecium verrucaria Bilirubin Oxidase Probed by Surface Plasmon Resonance, PMIRRAS, and Electrochemistry. Acs Catalysis 2016, 6 (8), 5482-5492.

5. Hitaishi, V. P.; Mazurenko, I.; Harb, M.; Clement, R.; Taris, M.; Castano, S.; Duche, D.; Lecomte, S.; Ilbert, M.; de Poulpiquet, A.; Lojou, E., Electrostatic-Driven Activity, Loading, Dynamics, and Stability of a Redox Enzyme on FunctionalizedGold Electrodes for Bioelectrocatalysis. Acs Catalysis 2018, 8 (12), 12004-12014.

6. Singh, K.; Blanford, C. F., Electrochemical Quartz Crystal Microbalance with Dissipation Monitoring: A Technique to Optimize Enzyme Use in Bioelectrocatalysis. Chemcatchem 2014, 6 (4), 921-929.

7. Vincent, K. A.; Parkin, A.; Armstrong, F. A., Investigating and exploiting the electrocatalytic properties of hydrogenases. Chemical Reviews 2007, 107 (10), 4366-4413.

8. Zigah, D.; Lojou, E.; de Poulpiquet, A., Micro- and Nanoscopic Imaging of Enzymatic Electrodes: A Review. ChemElectroChem 2019, 6 (22), 5524-5546.

9. Lei, R.; Stratmann, L.; Schaefer, D.; Erichsen, T.; Neugebauer, S.; Li, N.; Schuhmann, W., Imaging Biocatalytic Activity of Enzyme-Polymer Spots by Means of Combined Scanning Electrochemical Microscopy/Electrogenerated Chemiluminescence. Analytical Chemistry 2009, 81 (12), 50705074.

10. Zhao, F.; Conzuelo, F.; Hartmann, V.; Li, H.; Stapf, S.; Nowaczyk, M. M.; Rogner, M.; Plumere, N.; Lubitz, W.; Schuhmann, W., A novel versatile microbiosensor for local hydrogen detection by means of scanning photoelectrochemical microscopy. Biosensors \& bioelectronics 2017, 94, 433-437.

11. Martin, G. L.; Minteer, S. D.; Cooney, M. J., Spatial Distribution of Malate Dehydrogenase in Chitosan Scaffolds. Acs Applied Materials \& Interfaces 2009, 1 (2), 367-372.

12. Martin, G. L.; Minteer, S. D.; Cooney, M., Fluorescence characterization of immobilization induced enzyme aggregation. Chemical Communications 2011, 47 (7), 2083-2085.

13. Wu, F.; Minteer, S. D., Fluorescence Characterization of Co-immobilization-Induced Multi-Enzyme Aggregation in a Polymer Matrix Using Forster Resonance Energy Transfer (FRET): Toward the Metabolon Biomimic. Biomacromolecules 2013, 14 (8), 2739-2749.

14. Mateo-Mateo, C.; Michardiere, A.-S.; Gounel, S.; Ly, I.; Rouhana, J.; Poulin, P.; Mano, N., Wet-Spun Bioelectronic Fibers of Imbricated Enzymes and Carbon Nanotubes for Efficient Microelectrodes. Chemelectrochem 2015, 2 (12), 19081912.

15. Zhang, L.; Carucci, C.; Reculusa, S.; Goudeau, B.; Lefrancois, P.; Gounel, S.; Mano, N.; Kuhn, A., Rational Design of Enzyme-Modified Electrodes for Optimized Bioelectrocatalytic Activity. Chemelectrochem 2019, 6 (19), 4980-4984.

16. Engstrom, R. C.; Ghaffari, S.; Qu, H. W., FLUORESCENCE IMAGING OF ELECTRODE SOLUTION INTERFACIAL PROCESSES. Analytical Chemistry 1992, 64 (21), 2525-2529.

17. Bowyer, W. J.; Xie, J.; Engstrom, R. C., Fluorescence imaging of the heterogeneous reduction of oxygen. Analytical Chemistry 1996, 68 (13), 2005-2009.

18. Vitt, J. E.; Engstrom, R. C., Imaging of oxygen evolution and oxide formation using quinine fluorescence. Analytical Chemistry 1997, 69 (6), 1070-1076.

19. Bouffier, L.; Doneux, T.; Goudeau, B.; Kuhn, A., Imaging Redox Activity at Bipolar Electrodes by Indirect 
Fluorescence Modulation. Analytical Chemistry 2014, 86 (8), 3708-3711.

20. Guerrette, J. P.; Percival, S. J.; Zhang, B., Fluorescence Coupling for Direct Imaging of Electrocatalytic Heterogeneity. Journal of the American Chemical Society 2013, 135 (2), 855861.

21. Oja, S. M.; Zhang, B., Imaging Transient Formation of Diffusion Layers with Fluorescence-Enabled Electrochemical Microscopy. Analytical Chemistry 2014, 86 (24), 12299-12307.

22. Oja, S. M.; Guerrette, J. P.; David, M. R.; Zhang, B., Fluorescence-Enabled Electrochemical Microscopy with Dihydroresorufin as a Fluorogenic Indicator. Analytical Chemistry 2014, 86 (12), 6040-6048.

23. Djoumer, R.; Anne, A.; Chovin, A.; Demaille, C.; Dejous, C.; Hallil, H.; Lachaud, J.-L., Converting Any Faradaic Current Generated at an Electrode under Potentiostatic Control into a Remote Fluorescence Signal. Analytical Chemistry 2019, 91 (10), 6775-6782.

24. Bouffier, L.; Doneux, T., Coupling electrochemistry with in situ fluorescence (confocal) microscopy. Current Opinion in Electrochemistry 2017, 6 (1), 31-37.

25. Bizzotto, D., In situ spectroelectrochemical fluorescence microscopy for studying electrodes modified by molecular adsorbates. Current Opinion in Electrochemistry 2018, 7, 161-171.

26. Zhao, J.; Branagan, S. P.; Bohn, P. W., Single-Molecule Enzyme Dynamics of Monomeric Sarcosine Oxidase in a GoldBased Zero-Mode Waveguide. Applied Spectroscopy 2012, 66 (2), 163-169.

27. Zhao, J.; Zaino, L. P.; Bohn, P. W., Potential-dependent single molecule blinking dynamics for flavin adenine dinucleotide covalently immobilized in zero-mode waveguide array of working electrodes. Faraday Discussions 2013, 164, 57-69.

28. Zaino, L. P.; Grismer, D. A.; Han, D.; Crouch, G. M.; Bohn, P. W., Single occupancy spectroelectrochemistry of freely diffusing flavin mononucleotide in zero-dimensional nanophotonic structures. Faraday Discussions 2015, 184, 101115.

29. Pruchyathamkorn, J.; Yang, M.; Amin, H. M. A.; Batchelor-McAuley, C.; Compton, R. G., Imaging Electrode Heterogeneity Using Chemically Confined Fluorescence Electrochemical Microscopy. Journal of Physical Chemistry Letters 2017, 8 (24), 6124-6127.

30. Yang, M.; Batchelor-McAuley, C.; Katelhon, E.; Compton, R. G., Reaction Layer Imaging Using Fluorescence Electrochemical Microscopy. Analytical Chemistry 2017, 89 (12), 6870-6877.

31. Sentic, M.; Milutinovic, M.; Kanoufi, F.; Manojlovic, D.; Arbault, S.; Sojic, N., Mapping electrogenerated chemiluminescence reactivity in space: mechanistic insight into model systems used in immunoassays. Chemical Science 2014, 5 (6), 2568-2572.

32. Lefrancois, P.; Vajrala, V. S. R.; Arredondo, I. B.; Goudeau, B.; Doneux, T.; Bouffier, L.; Arbault, S., Direct oxidative pathway from amplex red to resorufin revealed by in situ confocal imaging. Physical Chemistry Chemical Physics 2016, 18 (37), 25817-25822.

33. Doneux, T.; Bouffier, L.; Goudeau, B.; Arbault, S., Coupling Electrochemistry with Fluorescence Confocal Microscopy To Investigate Electrochemical Reactivity: A Case Study with the Resazurin-Resorufin Fluorogenic Couple. Analytical Chemistry 2016, 88 (12), 6292-6300.

34. de Poulpiquet, A.; Goudeau, B.; Garrigue, P.; Sojic, N.; Arbault, S.; Doneux, T.; Bouffier, L., A snapshot of the electrochemical reaction layer by using 3 dimensionally resolved fluorescence mapping. Chemical Science 2018, 9 (32), 66226628 .

35. Mano, N.; de Poulpiquet, A., O-2 Reduction in Enzymatic Biofuel Cells. Chemical Reviews 2018, 118 (5), 23922468.

36. Xia, H.-q.; Kitazumi, Y.; Shirai, O.; Kano, K., Enhanced direct electron transfer-type bioelectrocatalysis of bilirubin oxidase on negatively charged aromatic compoundmodified carbon electrode. Journal of Electroanalytical Chemistry 2016, 763, 104-109.

37. Cracknell, J. A.; McNamara, T. P.; Lowe, E. D.; Blanford, C. F., Bilirubin oxidase from Myrothecium verrucaria: $\mathrm{X}$-ray determination of the complete crystal structure and a rational surface modification for enhanced electrocatalytic O-2 reduction. Dalton Transactions 2011, 40 (25), 6668-6675.

38. Sjoback, R.; Nygren, J.; Kubista, M., ABSORPTION AND FLUORESCENCE PROPERTIES OF FLUORESCEIN. Spectrochimica Acta Part a-Molecular and Biomolecular Spectroscopy 1995, 51 (6), L7-L21.

39. Paziewska-Nowak, A.; Raczynski, T.; Pijanowska, D. G.; Janczak, D.; Jakubowska, M.; Ieee, Evaluation of Fluorescein as a Label in Electrochemical and Optical Measurements. 2018.

40. Singh, K.; McArdle, T.; Sullivan, P. R.; Blanford, C. F., Sources of activity loss in the fuel cell enzyme bilirubin oxidase. Energy \& Environmental Science 2013, 6 (8), 2460-2464.

41. Lei, C.; Hu, D.; Ackerman, E. J., Single-molecule fluorescence spectroelectrochemistry of cresyl violet. Chemical Communications 2008, (43), 5490-5492. 


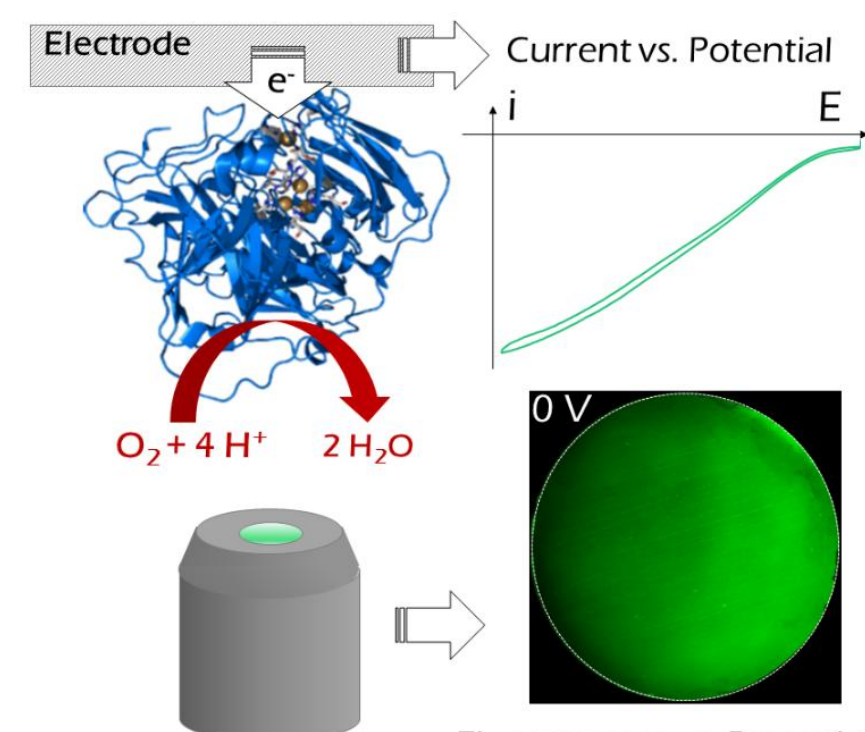

Fluorescence vs. Potential

Table of Contents 